\title{
LEVANTAMENTO FLORÍSTICO DE UMA MATA DECÍDUA EM UBERLÂNDIA, MINAS GERAIS, BRASIL ${ }^{1}$
}

\author{
Luciene Alves Rodrigues ${ }_{2}^{2}$ \\ Glein Monteiro de Araújo
}

Recebido em 05/05/97. Aceito em 31/12/97

\begin{abstract}
RESUMO - (Levantamento floristico de uma mata decídua em Uberlândia, Minas Gerais, Brasil). Esse trabalho consistiu de levantamento florístico em mata mesófila decídua situada em solo raso de origem basáltica em Uberlândia, Minas Gerais. Foram feitas visitas quinzenais à mata, no periodo de fevereiro/93 a junho/95, para coletas de exemplares botânicos férteis. O material coletado foi incorporado ao Herbário da Universidade Federal de Uberlândia (HUFU). Foram identificadas 105 espécies de árvores, arbustos, lianas e herbáceas, distribuidas em 88 gêneros e 43 familias. As espécies mais freqüentes na mata foram: Anadenanthera colubrina, Bauhinia sp., Croton sp., Myracrodruon urundeuva e Sweetia fruticosa. Euphorbiaceae (10), Rubiaceae (8), Mimosaceae (6), Myrtaceae (6), Caesalpinaceae (5) e Fabaceae (5) foram as familias com maior número de espécies identificadas.
\end{abstract}

Palavras-chave: Florística, mata decídua, Minas Gerais, Brasil

\begin{abstract}
Floristc survey of an area of deciduous forest in Uberlândia, Minas Gerais, Brazil). A floristic survey was carried out in a deciduous forest on a shallow basaltic soil, in Uberlândia, Minas Gerais. Visits were performed fortnightly from February/93 to June/95. The sampled material was lodged at the Herbarium of the Universidade Federal de Uberlândia (HUFU). A list is provided with 105 species of trees, shrubs, scandent shrubs, lianas and herbs, belonging to 88 genera and 43 botanic families. The most frequent species were: Anadenanthera colubrina, Bauhinia sp., Croton sp., Myracrodruon urundeuva and Sweetia fruticosa. Euphorbiaceae (10), Rubiaceae (8), Mimosaceae (6), Myrtaceae (6), Caesalpinaceae (5) e Fabaceae (5) were the families with the largest numbers of identified species.
\end{abstract}

Key words: Floristc, deciduous forest, Minas Gerais, Brazil.

\section{Introdução}

A região do cerrado foi uma das que mais ampliou a produção agrícola nos últimos anos. Isso fez com que a vegetação natural fosse cada vez mais reduzida e a perpetuação de algumas espécies vegetais e animais, ameaçada (Wagner 1985). Nessa região, além do cerrado (sentido amplo) encontram-se outras formações vegetais,

\footnotetext{
'Trabalho apresentado no XLVI Congresso Nacional de Botânica, Ribeirão Preto, SP. Projeto de iniciação cientifica, processo 178/94 (PIBIC/CNPq/UFU)

2 Universidade Federal de Uberlândia, Departamento de Biociências, CEP 38400-902, Uberlândia, MG
} 
como as matas mesófilas, que se diferenciam, principalmente, pelas condições edáficas. Essas matas ocorrem em forma de manchas, variando a deciduidade, de acordo com a disponibilidade de água dos solos em que se encontram (Rizzini 1979).

No Brasil Central, as matas decíduas ocorrem em solos rasos, em afloramentos de calcário e outras rochas básicas e, em geral, apresentam baixa similaridade florística com as matas semidecíduas e perenifólias (Ratter et al. 1978; Eiten 1983).

Segundo Alho \& Martins (1995), para "a conservação da diversidade biológica em seu senso mais específico", é necessário que as unidades de conservação do cerrado incluam áreas ainda mal representadas, como as matas decíduas em afloramentos rochosos.

Para que se possa estabelecer modelos de conservação e manejo das áreas remanescentes e recuperar áreas perturbadas, devem ser realizados estudos amplos das espécies vegetais que ocorrem nesses ecossistemas. Um dos pontos de partida para realizar tais estudos é conhecer a composição florística dessas comunidades.

O presente trabalho teve como objetivo realizar levantamento florístico em uma mata decídua situada, em solo raso de origem basáltica, no município de Uberlândia, MG.

\section{Material e métodos}

A área de estudo localiza-se na Fazenda Buriti, próxima ao vale do Rio Araguari, Uberlândia, Estado de Minas Gerais. A mata possui cerca de 10ha e está situada em solo raso, de origem basáltica, com declividade acentuada. O clima de Uberlândia, segundo a classificação de Köppen, é do tipo Aw, megatérmico, com chuvas de verão e seca de inverno; a temperatura média anual é de $22^{\circ} \mathrm{C}$, com um total pluviométrico de $1500 \mathrm{~mm} /$ ano (Rosa et al. 1991).

No período de fevereiro/93 a junho/95, foram realizadas visitas quinzenais à mata para coleta de material botânico fértil. Esse trabalho foi realizado ao longo de trilhas, em toda a extensão da comunidade vegetal e em suas proximidades, onde a mata havia sido alterada.

Todo o material coletado foi levado para o Herbário da Universidade Federal de Uberlândia (HUFU) e herborizado de acordo com métodos descritos por Fidalgo \& Bononi (1984). A identificação dos exemplares foi realizada utilizando-se literatura disponível, por comparação com exsicatas dos Herbários da UFU e UnB, e/ou por especialistas. O sistema de classificação das famílias adotado foi o de Cronquist (1981).

\section{Resultados e discussão}

Foram identificadas 105 espécies (69 arbóreas, 12 arbustivas, 3 arbustivas escandentes, 6 lianas e 15 herbáceas), distribuídas em 88 gêneros e 43 famílias (Tab. 1). Entre as árvores de grande porte encontradas na mata, predominam Anadenanthera colubrina, Myracrodruon urundeuva e Sweetia fruticosa, e no subosque, Bauhinia sp., Celtis iguanaea, Chomelia sericea, Croton sp. e outras espécies da familia Euphorbiaceae.

Euphorbiaceae (10), Rubiaceae (8), Mimosaceae (6), Myrtaceae (6), Fabaceae (5) e Caesalpinaceae (5) foram as famílias com maior número de espécies identificadas. 
TABELA 1. Lista de espécies identificadas da mata decídua da Fazenda Buriti, Uberlândia-MG. (a =árvore; b $=$ arbusto $; \mathrm{c}=$ arbusto escandente $; \mathrm{d}=$ liana; $\mathrm{e}=$ herbácea $)$.

\begin{tabular}{|c|c|c|}
\hline Familia/espécie & Hábito & Nome popular \\
\hline \multicolumn{3}{|l|}{ ACANTHACEAE } \\
\hline Laphostachys floribunda Pohl & $\mathrm{e}$ & cafezinho \\
\hline Ruellia brevifolia (Pohl) Ezcurra & $\mathrm{e}$ & pingo-de-sangue \\
\hline \multicolumn{3}{|l|}{ ANACARDIACEAE } \\
\hline Myracrodruon urundeuva Allemão & a & aroeira, urundeúva, \\
\hline \multicolumn{3}{|l|}{ APOCYNACEAE } \\
\hline Aspidosperma cuspa (H.B.K.) S.F. Blake & a & tambú-branco \\
\hline \multicolumn{3}{|l|}{ ARALIACEAE } \\
\hline Sciadodendron excelsum Griseb. & a & carobão, lagarto, sabugueiro \\
\hline \multicolumn{3}{|l|}{ ASTERACEAE } \\
\hline Cosmos caudatus H.B.K. & $\mathrm{e}$ & picão \\
\hline Dasyphyllum synacanthum (Baker) Cabrera & $\mathrm{c}$ & espinho-de-agulha \\
\hline Eupatorium pictum Gardner & b & \\
\hline Vernonea ferruginea Less & $\mathrm{b}$ & assa-peixe \\
\hline \multicolumn{3}{|l|}{ BIGNONIACEAE } \\
\hline Arrabidea florida DC. & c & cipó-neve \\
\hline Macfadyena unguis-cati (L.) Gentry & d & unha-de-gato \\
\hline Tabebuia roseo-alba (Ridley) Sandw. & a & ipê \\
\hline \multicolumn{3}{|l|}{ BOMBACACEAE } \\
\hline Chorisia speciosa St.Hil. & a & paineira, barriguda \\
\hline \multicolumn{3}{|l|}{ BORAGINACEAE } \\
\hline Cordia alliodora Cham. & a & chá-de-bugre \\
\hline Tournefortia paniculata Cham . & $\mathrm{d}$ & cipó-mulato \\
\hline \multicolumn{3}{|l|}{ CAESALPINACEAE } \\
\hline Bauhinia sp. & a & unha-de-cabrito \\
\hline Cassia multijuga Rich. & a & pau-cigarra, aleluia \\
\hline Chamaecrista cf. flexuosa (L.) Greene & $\mathrm{e}$ & amarelinho \\
\hline Hymenaea courbaril L. & a & jatobá \\
\hline Senna pendula (Wiild.) Irwin \& Barneby & $\mathrm{e}$ & fedegoso \\
\hline \multicolumn{3}{|l|}{ CARICACEAE } \\
\hline Carica sp. & $\mathrm{b}$ & mamãozinho \\
\hline \multicolumn{3}{|l|}{ CELASTRACEAE } \\
\hline Maytenus floribunda Reisseck & a & erva-de-lagarto \\
\hline \multicolumn{3}{|l|}{ COMBRETACEAE } \\
\hline Terminalia brasiliensis Eichler & a & capitão-garrote \\
\hline \multicolumn{3}{|l|}{ COMMELINACEAE } \\
\hline Dichorisandra sp. & $\mathrm{e}$ & cana-de-macaco \\
\hline \multicolumn{3}{|l|}{ ERYTHROXYLACEAE } \\
\hline Erythroxylum cf. daphnites Mart. & a & fruto-de-pomba \\
\hline Erythroxylum aff. subracemosum Turcz. & a & fruto-de-tucano \\
\hline \multicolumn{3}{|l|}{ EUPHORBIACEAE } \\
\hline Acalypha gracilis Sprengel & b & acalifa \\
\hline Acalypha villosa Jacq. & b & urtiga-grande, urtigão \\
\hline Croton glandulosus L. & $\mathrm{e}$ & capichingui \\
\hline Croton gracilipes Baillon & b & sangue-de-dragão \\
\hline Croton sp. & a & mameleiro-preto \\
\hline Euphorbia heterophylla L. & $\mathrm{e}$ & leiteira \\
\hline Julocroton triqueter (Lam.) Didr. & b & crotom \\
\hline Manihot anomala Pohl & a & mandioca-brava \\
\hline Phyllanthus acuminatus Vahl & a & conambi \\
\hline Phyllanthus sp. & b & sabugueirinho \\
\hline
\end{tabular}


FABACEAE

Desmodium cf. barbatum (L.) Benth. Lonchocarpus cultratus (Vell.) H.C.Lima Machaerium cf. paraguariense Hassler Machaerium stipitatum (DC.)Vog.

Sweetia fruticos $a$ Sprengel

FLACOURTIACEAE

Casearia gossypiosperma Briquet

Casearia mariquitensis H.B.K.

Casearia sylvestris $\mathrm{Sw}$.

Prockia crucis P. Browne ex Lin

LECYTHIDACEAE

Cariniana estrellensis (Raddi) Kuntze

MALPIGHIACEAE

Byrsonima sp.

MARANTACEAE

Calathea sp.

MELIACEAE

Trichilia catigua A. Juss.

Trichilia elegans A. Juss.

Trichilia lagoensis C.DC.

MIMOSACEAE

Acacia cf. glomerosa Benth.

Acacia polyphylla Benth.

Albizia niopoides (Spruce ex Benth.) Burkart

Anadenathera colubrina (Vell.) Brenan

Enterolobium contortisiliquum (Vell.) Morong.

Piptadenia gonoacantha (Mart.) Macbr.

MORACEAE

Ficus enormis (Mart. e Miq.) Miquel.

Maclura tinctoria (L.) D. Don ex Steud.

MYRSINACEAE

Rapanea umbellata (Mart.) Mez.

MYRTACEAE

Calycorectes psidiiflorus (O. Berg.) Sobral

Campomanesia velutina Berg

Eugenia ligustrina (Sw.) Willd.

Eugenia cf. stictosepala Kiaerskov

Eugenia florida DC.

Myrcia tomentosa (Aublet) DC.

NYCTAGINACEAE

Guapira areolata (Heimerl) Lundell

OPILIACEAE

Agonandra brasiliensis Miers

POACEAE

Olyra ciliatifolia Raddi

Panicum trichoides Sw.

Setaria cf. vulpiseta (Lam.) Roem \& Schult.

POLYGONACEAE

Coccoloba mollis Casar.

PORTULACACEAE

Talinum patens (Jacq.) Willd.

RHAMNACEAE

Gouania aff. virgata Reisseck

Rhamnidium elaeocarpum Reisseck barbadinho

rabo-de-macaco

cateretê,jacarandá-branco

sapuva, sapuvinha

canjica, angelim

lingua-de-teiú, pau-de-espeto limãozinho

guaçatonga, erva-de-lagarto maçãzinha

jequitibá-branco, bingueiro

murici

maranta

catiguá

catiguá

goiaba-do-mato

munjolo

munjolo

camisa-fina, farinha-seca

angico

tamboril

pau-jacaré

gameleira, figueira

amoreira, tatajiba

pororoca

guaviroba-cabeluda guamirim

araçá

pimenteira

goiabeira-brava

maria-mole

quina-da-mata

pau-jaú, canudo

serralha 
RUBIACEAE

Alibertia sessilis (Vell.) K. Schum.

Chomelia pohliana Muell. Arg.

Chomelia sericea $\mathrm{M}$. Arg.

Coutarea hexandra (Jacq.) K. Schum.

Guettarda sp.

Genipa americana L.

Malanea cf. macrophylla Griseb.

Simira cf. viridifolia Steyerm.

RUTACEAE

Pilocarpus pinnatifolius Lam.

Zanthoxylum rhoifolium Lam.

SAPINDACEAE

Allophyllus sericeus (Camb.) Radlk.

Dilodendron bipinnatum Radlk.

Matayba cf. elaeagnoides Radlk.

Serjanea sp.

\section{SAPOTACEAE}

Chrysophyllum gonocarpum (Mart. \& Eichler) Engl.

Chrysophyllum marginatum (Hook. \& Arn.) Radlk.

Pouteria gardineri (Mart. \& Miq.) Baehni

Pouteria hispida Eyma

SOLANACEAE

Solanum sp.

STERCULIACEAE

Guazuma ulmifolia Lam.

Sterculia striata St.Hil. et Naud.

THEOPHRASTACEAE

Clavija integrifolia $\mathrm{M}$.

ULMACEAE

Celtis iguanaea (Jacq.) Sargent

Trema micrantha (L.) Blume

URTICACEAE

Urera caracasana (Jacq.) Gaudich ex Griseb.

VERBENACEAE

Aloysia virgata A.Juss.

Lantana aff. nivea Vent.

Lantana sp.

Petrea subsserrata Cham.

VIOLACEAE

Hybanthus sp.

VITACEAE

Cissus simsiana Roemer \& Schultes marmelada-de-cachorro veludo

fruto-de-passarinho veludo-preto

veludo-branco genipapo melanea

guatambu-bravo

jaborandi mamica-de-porca

três-folhas-vermelho maria-pobre, pau-pobre cambuatá-branco cipó-pau

guapeva guatambú-de-leite azeitona guapeva

jurubeba-brava

mutamba amendoim-de-cutia

fruta-de-cascavel

grão-de-galo, esporão candiúva, pau-de-fogo

urtigão, urtiga-brava

pau-lixa, lixa, lixeira camará camará coroa-de-viúva

\section{Discussão}

Entre as espécies encontradas, três foram citadas por Prado \& Gibbs (1993) como de ampla distribuição em áreas tropicais; são elas: Aspidosperma cuspa, Enterolobium contortisiliquum e Myracrodruon urundeuva. Esses autores consideraram Hymenaea courbaril, Coutarea hexandra e Cordia alliodora como tipos 
complexos e com tolerância ampla, pois ocorrem tanto em habitats muito úmidos, na Amazônia, quanto em locais secos, como a Caatinga.

Maclura tinctoria, Trema micrantha e Zanthoxylum rhoifolium ocorrem em todo o país, em várias formações florestais. Outras com ampla distribuição no Brasil são: Agonandra brasiliensis (PI, CE, GO, MG), Alibertia sessilis (CE, MT, GO, MG), Allophyllus sericeus (SP, RJ, MG, GO, BA), Anadenanthera colubrina (em solos férteis do MA, Nordeste do país, SP, MG, MS), Casearia gossypiosperma (PA até o PR), Dilodendron bipinnatum (MG, GO, MT, MS e SP, e ainda na Bolívia e no Peru), Guazuma ulmifolia (AM até PR), Machaerium stipitatum (RJ, SP, MG, MS até o RS), Piptadenia gonoacantha (RJ, MG, MS até SC), Pilocarpus pinnatifolius (de PE até SC), Sterculia striata (Sul da BA, ES, RJ, SP) e Sweetia fruticosa (Sul da BA, SP, MG, GO, MS e PR). As espécies Ficus enormis, Rhamnidium elaeocarpum, Machaerium paraguariense e Urera caracasana são mais comuns nas regiões Sudeste e Centro-Oeste (Correa 1975; Lorenzi 1992).

$\mathrm{O}$ fato de algumas espécies ocorrerem em diferentes formações vegetais, como mata de galeria e matas mesófilas decíduas, com diferentes disponibilidade de água ao longo do ano, pode não ser devido apenas à sua ampla valência ecológica, mas também à existência de microambientes dentro dessas comunidades, que as permitam estabelecerem. Além disso, características de pioneiras, como crescimento rápido e grande produção de sementes, segundo Prado \& Gibbs (1993), também pode explicar a ampla distribuição de algumas espécies.

Estudos do padrão de distribuição atual de mais de 80 táxons que ocorrem em várias áreas da América do Sul, e estudos palinológicos no Brasil Central, sugerem que o padrão de distribuição atualmente fragmentado de matas sazonais é vestígio de uma única e extensa formação contínua que, talvez, tenha atingido sua extensão máxima durante um período de clima seco e frio do Pleistoceno, coincidindo com o encolhimento das florestas úmidas (Ledru 1993; Prado \& Gibbs 1993). Algumas espécies, pertencentes a essa extensa formação, atravessariam o Brasil Central ocupando solos ricos em cálcio dentro dos cerrados (Prado \& Gibbs 1993).

Para Leonardos et al. (1994), além de estarem ligadas a refúgios de florestas, as manchas de vegetações mesófilas que ocorrem na região do cerrado estão relacionadas, principalmente, ao tipo de rocha que deu origem ao solo sob essas comunidades vegetais. A composição florística, de forma geral, é influenciada pelas propriedades químicas do solo, pela topografia, por microambientes (como clareiras e áreas em diferentes estádios de sucessão) e ainda, pela vegetação das áreas adjacentes (Pagano \& Leitão Filho 1987; Rodrigues et al. 1989; Cesar \& Leitão Filho 1990). Segundo Borchert (1994), a água é o principal fator ambiental que influencia na distribuição de espécies de matas secas.

As dez principais famílias possuem $55,5 \%$ das espécies identificadas. Euphorbiaceae contribuiu com o maior número, fato que não ocorreu em outros trabalhos em matas do Triângulo Mineiro, como os de Araújo (1992) e Schiavini (1992). Mimosaceae, Myrtaceae e Rubiaceae, ao contrário, estão entre as principais no presente trabalho e nos anteriormente citados, demonstrando ampla distribuição regional. Segundo Gentry (1995), Euphorbiaceae é a familia mais bem representada em matas decíduas neotropicais apresentando, em geral, espécies arbustivas. 
Lauraceae que, geralmente, ocorre com muitas espécies em mata de galeria e matas mesófilas semidecíduas do Estado de São Paulo e Minas Gerais (Araújo 1992; Rodrigues et al. 1992; Schiavini 1992; Kotchetkoff-Henriques \& Joly 1994; Oliveirafilho et al. 1994), não foi encontrada na mata decídua da Fazenda Buriti. Melastomataceae e Vochysiaceae também não foram verificadas na mata estudada. Segundo Goodland (1979), essas duas famílias ocorrem com numerosas espécies em cerrado do Triângulo Mineiro, são acumuladoras de alumínio e bem adaptadas a solos pobres em nutrientes. O solo raso e com alta fertilidade natural, da mata do presente estudo (Araújo et al. 1997), pode ter contribuído para a ausência de espécies das famílias Melastomataceae e Vochysiaceae.

O conhecimento da flora é de fundamental importância em programas de preservação ambiental para remanescentes, como a mata decídua da Fazenda Buriti. As matas mesófilas decíduas, por serem fragmentadas, são de dificil interpretação, sendo necessários estudos mais amplos para relacionar os efeitos dessa fragmentação na composiçào florística dessas comunidades. A conservação desses remanescentes é necessária e urgente, pois esses ecossistemas estão cada vez mais ameaçados pela ação antrópica.

\section{Referências bibliográficas}

Alho, C.J.R. \& Martins, E.S. 1995. De grão em grão o Cerrado perde espaço (Cerrado - Impactos do Processo de Ocupação). Brasília. WWF.

Araújo, G.M. 1992. Comparação da estrutura e do teor de nutrientes nos solos e nas folhas de espécies arbóreas de duas matas mesófilas semidecíduas no Triângulo Mineiro. Campinas. Universidade Estadual de Campinas. Tese de Doutorado.

Araújo, G.M.; Rodrigues, L.A. \& Ivizi, L. 1997. Estrutura fitossociológica e fenologia de espécies lenhosas de mata decidua em Uberlândia, MG. p. 22-28 In: L.L., Leite \& C.H., Saito (ed.), Contribuição ao conhecimento ecológico do cerrado. Brasilia. UnB.

Borchert, R. 1994. Site water availability and stem water storage determine water status, phenology and distribution of trees in a tropical dry forest in Costa Rica. Ecology 75: 1437-1449.

Cesar, O. \& Leitão Filho, H.F. 1990. Estudo florístico quantitativo de mata mesófila semidecidua na Fazenda Barreiro Rico, Municipio de Anhembi, São Paulo. Revista Brasileira Biologia, 50: 133-147.

Corrêa, M.P. 1975. Dicionário das plantas úteis do Brasil e das exóticas cultivadas. Rio de Janeiro. IBDF, Ministério da Agricultura. v. 1-6.

Cronquist, A. 1981. An integral system of classification of flowering plants. New York. Columbia University Press.

Eiten, G. 1983. Classificação da vegetação do Brasil. Brasília. CNPq/Coordenação Editorial.

Fidalgo, O. \& Bononi, V.L.R. (coord.) 1984. Técnicas de coleta, preservação e herborização de material botânico. São Paulo. Instituto de Botânica.

Gentry, A. H. 1995. Diversity and floristic composition of neotropical dry forests. In: S.H. Bullock, H.A. Mooney, \& E. Medina (ed.), Seasonally dry tropical forests. New York. Cambridge University Press, p. 146-194.

Goodland, R. 1979. Análise ecológica da vegetação do Cerrado. p. 61-162. In: R., Goodland \& M.G., Ferri Ecologia do Cerrado, São Paulo. EDUSP.

Kotchetkoff-Henriques, O. \& Joly, C.A. 1994. Estudo florístico e fitossociológico em uma mata mesófila semidecídua da Serra de Itaqueri, Estado de São Paulo, Brasil . Revista Brasileira Biologia 54: 477-487.

Ledru, M.P. 1993. Late quaternary environmental and climate changes in Central Brazil. Quaternary Research, 39: $90-98$.

Leonardos, O.H.; Martins, E.S. \& Santos, R.V. 1994. Lithospheric constraints of cerrado and rainforest ecological systems. Anais Academia Brasileira de Ciências, 66: 167-172.

Lorenzi, H. 1992. Árvores brasileiras: manual de identificação e cultivo de plantas arbóreas nativas do Brasil. Nova Odessa. Plantarum. 
Oliveira-Filho, A.T.; Almeida, R.J.; Mello, J.M. \& Gavilanes, M.L. 1994. Estrutura fitossociológica e variáveis ambientais em um trecho de mata ciliar do córrego dos Vilas Boas, Reserva Biológica do Poço Bonito, Lavras (MG). Revista Brasileira de Botânica 17: 67-85.

Pagano, S.N. \& Leitão Filho, H.F. 1987. Composição florística do estrato arbóreo de mata mesófila semidecidua, no Municipio de Rio Claro (Estado de São Paulo). Revista Brasileira de Botânica 10: 37-47.

Prado, D.E. \& Gibbs, P.E. 1993. Patterns of species distributions in the dry seasonal forests of South America. Annals of the Missouri Botanical Garden, 80: 902-927.

Ratter, J.A., Askew, G.P., Montgomery, R.F. \& Gifford, D.R. 1978. Observations on forests of some mesotrophic soils in Central Brazil. Revista Brasileira de Botânica 1: 47-58.

Rizzini, C.T. 1979. Tratado de fitogeografia do Brasil, aspectos sociológicos e florísticos. São Paulo. EDUSP. v.2.

Rodrigues, R.R.; Morellato, L.P.C.; Joly, C.A. \& Leitão Fliho, H.F. 1989. Estudo florístico e fitossociológico em um gradiente altitudinal de mata estacional mesófila semidecídua, na Serra do Japi, Jundiai, SP. Revista Brasileira de Botânica 12: 71-84.

Rosa, R.; Lima, S.C. \& Assunção, W.L. 1991. Abordagem preliminar das condições climáticas de Uberlândia (MG). Sociedade \& Natureza 3: 91-108.

Schiavini, I. 1992. Estrutura das comunidades arbóreas de mata de galeria da Estação Ecológica do Panga (Uberlândia, MG). Campinas. Universidade Estadual de Campinas. Tese de Doutorado.

Wagner, E. 1985. Desenvolvimento da região dos cerrados. p.19-31. In: Goedert, W.J. (ed.), Solos dos Cerrados: tecnologias e estratégias de manejo. Brasilia. EMBRAPA. 